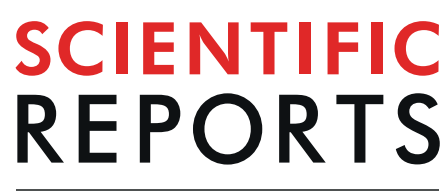

natureresearch

Received: 12 March 2019

Accepted: 1 August 2019

Published online: 19 August 2019

\section{Engineering a Polyspecific Pyrrolysyl-tRNA Synthetase by a High Throughput FACS Screen}

\author{
Adrian Hohl1,2, Ram Karan ${ }^{1}$, Anastassja Akal ${ }^{1,2}$, Dominik Renn ${ }^{1,2}$, Xuechao Liu ${ }^{1}$, \\ Seema Ghorpade $\mathbb{D}^{1}$, Michael Groll ${ }^{2}$, Magnus Rueping ${ }^{1}$ \& Jörg Eppinger $\mathbb{1}^{1}$
}

The Pyrrolysyl-tRNA synthetase (PyIRS) and its cognate tRNA ${ }^{\text {Pyl }}$ are extensively used to add noncanonical amino acids (ncAAs) to the genetic code of bacterial and eukaryotic cells. However, new ncAAs often require a cumbersome de novo engineering process to generate an appropriate PyIRS/ tRNA ${ }^{\text {Pyl }}$ pair. We here report a strategy to predict a PyIRS variant with novel properties. The designed polyspecific PyIRS variant HpRS catalyzes the aminoacylation of 31 structurally diverse ncAAs bearing clickable, fluorinated, fluorescent, and for the first time biotinylated entities. Moreover, we demonstrated a site-specific and copper-free conjugation strategy of a nanobody by the incorporation of biotin. The design of polyspecific PyIRS variants offers an attractive alternative to existing screening approaches and provides insights into the complex PyIRS-substrate interactions.

Site-specific incorporation of non-canonical amino acids (ncAAs) is a powerful tool to implement novel functions into the proteome ${ }^{1-5}$. The central element of a selective orthogonal ncAA incorporation system is an aminoacyl-tRNA synthetase/tRNA (aaRS/tRNA) pair that does not cross-react with the original aaRSs, tRNAs, or canonical amino acids of the host cell ${ }^{6}$. The pyrrolysyl-tRNA synthetase/tRNA ${ }^{\text {Pyl }}$ pair (PylRS/tRNA ${ }^{\text {Pyl }}$ ) has become a popular choice for the genetic code expansion ${ }^{7,8}$. This success is mainly attributed to the substrate flexibility of the active site $e^{9,10}$, the orthogonality in prokaryotic and eukaryotic cells ${ }^{11}$, and the inherent suppression of the amber codon ${ }^{8}$. Although over $150 \mathrm{ncAAs}$ were genetically encoded during the past decade, these structures were mainly based on few core motifs ${ }^{5}$. Several structural functionalities still remain elusive, e.g., biotinylated ncAAs. The main factor limiting further expansion of the genetic code is the engineering of suitable aaRS/tRNA pairs. This process requires the construction of large mutant libraries from which all non-functional and non-orthogonal aaRS variants are eliminated through alternating rounds of positive and negative selection $^{2}$. In most cases, selection screens are carried out as dead-and-alive assays on agar plates ${ }^{2,12,13}$. However, the selection conditions of this approach are hardly tunable, and therefore a beneficial aaRS might be accidentally eliminated ${ }^{1,6,14}$.

Screens based on fluorescence-activated cell sorting (FACS) provide a more sensitive and quantitative read-out and have been successfully applied to evolve the aaRS specificity ${ }^{15-18}$. However, the reported experimental setups involve several negative and positive selection rounds, resulting in a cumbersome process ${ }^{17}$.

Extensive efforts have been made to create a vast number of aaRS in the last years. These synthetases are often selected and used for the incorporation of a particular ncAA, although many engineered synthetases are polyspecific and aminoacylate a range of different ncAAs ${ }^{19,20}$. Hence, the aaRS-substrate information is not fully exploited and may be used to incorporate novel ncAAs, for which the de novo selection process had failed.

The current study investigated, whether the structural features of different PylRS variants can be recombined to a new synthetase exhibiting inherited and novel properties. Therefore, we created a mutant library based on reported PylRS variants and applied a sensitive selection screen. The identified PylRS-ncAA pairs provided insights for the prediction of the "highly polyspecific pyrrolysyl-tRNA synthetase" (HpRS). HpRS showed a tailored substrate scope and genetically incorporated for the first time biotin lysine. Furthermore, we demonstrate three different conjugation strategies of a GFP-nanobody using HpRS.

${ }^{1}$ King Abdullah University of Science and Technology (KAUST), KAUST Catalysis Center (KCC), Physical Sciences and Engineering Division (PSE), Thuwal, 23955-6900, Saudi Arabia. ${ }^{2}$ Technical University of Munich (TUM), Center for Integrated Protein Science Munich in the Department Chemistry, Garching, Germany. Adrian Hohl and Ram Karan contributed equally. Correspondence and requests for materials should be addressed to R.K. (email: ram.karan@ kaust.edu.sa) or M.R. (email: magnus.rueping@kaust.edu.sa) or J.E. (email: jorg.eppinger@kaust.edu.sa) 

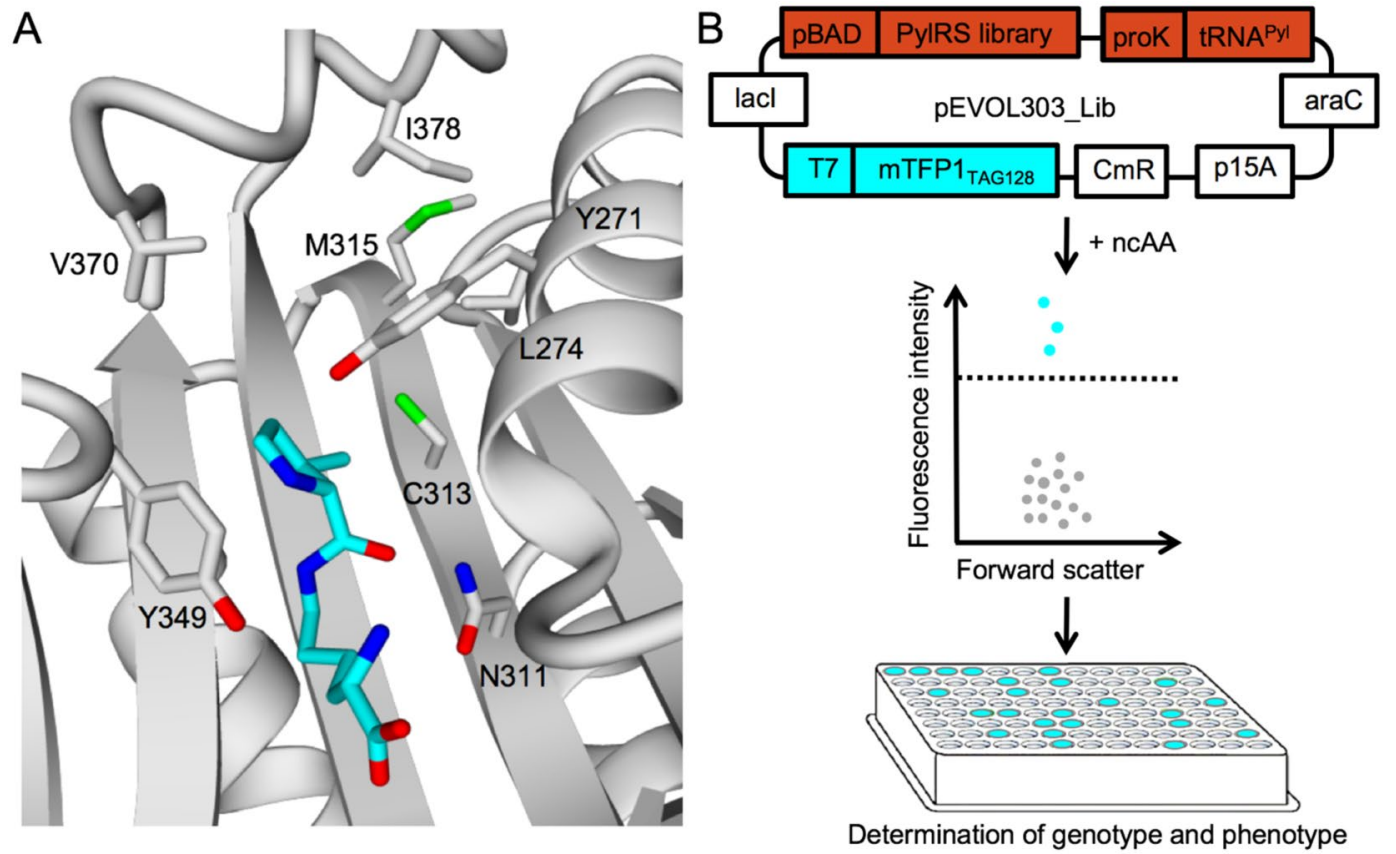

Figure 1. (A) Homology model of PylRS in complex with pyrrolysine (cyan) in the binding pocket. (B) Cells transformed with the plasmid pEVOL303_Lib are screened for ncAA incorporation by mTFP1-based fluorescence intensity and forward scatter using FACS. Fluorescent cells (cyan) exceeding a set threshold are sorted separately from nonfluorescent cells (grey) for sequencing on a 96-well plate. pEVOL303_Lib encodes for the PylRS library, tRNA ${ }^{\text {Pyl }}$ and, mTFP1 $_{\text {TAG128. }}$ p15A origin of replication (p15A), araC repressor gene (araC), chloramphenicol acetyltransferase marker (CmR), lac repressor (lacI), T7 promoter (T7), proK promoter (proK), araBAD promoter (pBAD).

\section{Results and Discussion}

Library design. First, we designed a mutant library based on existing PylRS variants and a homology model of the Methanosarcina barkeri (M. barkeri) PylRS (Fig. 1A). The mutation Y349F enhanced the aminoacylation efficiency of PylRS and was included in the mutant library by default ${ }^{10}$. We maintained the orthogonality of the active site by retaining position $\mathrm{N} 311$ to eliminate a negative selection ${ }^{21,22}$. Based on these structural considerations, we selected the six amino acids Y271, L274, C313, M315, V370, and I378 to create a mutant PylRS library (Supplementary Table 1). In particular, smaller residues were inserted at these positions to increase the volume of the pocket and to allow the aminoacylation of larger ncAAs.

Screening of PyIRS variants. Dead-and-alive assays are the preferred method for screening large quantities of variants in a short time as demonstrated on existing aaRS/tRNA pairs ${ }^{2,13}$. Nevertheless, this screening method suffers from a harsh and hardly tunable selection pressure, causing slow cell growth and low survival rates ${ }^{17}$. Consequently, even beneficial variants might be unintentionally excluded from the library. Unlike dead-and-alive assays, fluorescence-activated cell sorting (FACS) provides a tunable sorting stringency over a broad dynamic range for the ncAA incorporation ${ }^{15,17,23}$.

To set up a fast FACS screen, the PylRS library, tRNA ${ }^{\text {Pyl }}$ and the cyan monomeric teal fluorescent protein 1 (mTFP1) were expressed from a single plasmid (Fig. 1B). The mTFP1 contained an amber codon at position 128 $\left(\mathrm{mTFP}_{\mathrm{TAG} 128}\right)$ and allowed to quantify the incorporation efficiency of a ncAA according to the fluorescence intensity. E. coli cells transformed with pEVOL303_Lib were grown both in the absence and presence of ncAAs 1-23 (Fig. 2).

Then, the forward scatter and fluorescent signals of each cell were analysed by FACS. In the presence of ncAA 1, several cells depicted significant fluorescent signals (Fig. 3A, right plot) and negligible fluorescence in the absence of ncAAs indicates the orthogonality of the mutant library (Fig. 3A, left plot). Notably, the fluorescent cells appeared to be a heterogeneous population, suggesting that multiple PylRS variants enabled the aminoacylation of ncAA 1. All fluorescent cells exceeding a set threshold were sorted on a 96-well plate supplied with conditioned medium, recovered overnight, and the PylRS variants were sequenced. The medium contained cell-secreted growth factors that improved the survival rate of single cells three-fold compared to the untreated lysogeny broth (LB) medium (Fig. 3B). In the case of ncAA 1, seven different PylRS variants facilitated its incorporation (Fig. $3 \mathrm{C}$ ). Next, expression of $\mathrm{mTFP}_{\mathrm{TAG} 128}$ was repeated in the presence of the sequenced PylRS/tRNA ${ }^{\mathrm{Pyl}}$ pairs and ncAA 1 to analyze the incorporation efficiency and orthogonality of the different variants (Fig. 3C).

Overall, the screen identified 151 PylRS variants (110 unique and 41 redundant) that incorporated 20 out of the 23 initially tested ncAAs. The incorporation of ncAAs 9, 22, and 23 were not detected by FACS. Seventeen PylRS variants accepted multiple ncAAs. Individual ncAAs were aminoacylated by up to 16 different PylRS variants (Supplementary Table 3). The amino acid abundancies of the six mutated positions of all sorted PylRS 

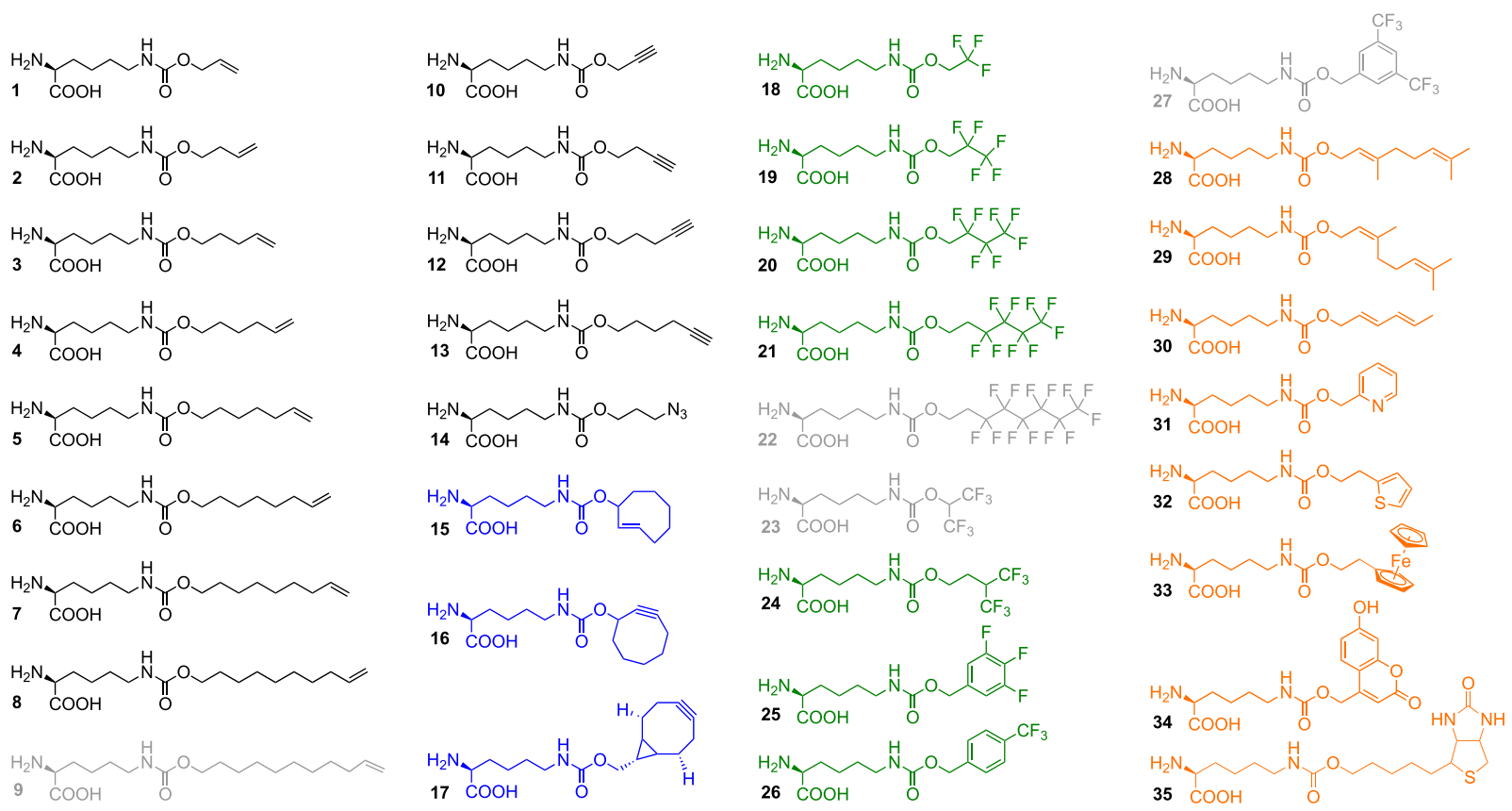

Figure 2. Chemical structures of analyzed ncAAs. Class I: linear alkenes, alkynes, and azide (black). Class II: cyclic alkenes and alkynes (blue). Class III: fluorinated side chains (green). Class IV: diverse functionalities (orange). Grey ncAA structures were rejected.

variants are summarized for each ncAA in a heat map (Fig. 4). We divided the tested ncAAs 1-23 into three structural classes to elucidate key mutations in the binding pocket. Class I ncAAs 1-8 (alkenes) and 10-14 (alkynes) bear a linear aliphatic tail. Like a molecular ruler, these ncAAs allow to assess the maximum carbon chain length accepted by our PylRS library. Class II ncAAs 15-17 are cyclic and can be used in strain-promoted cycloadditions $^{24,25}$. Highly fluorinated side chains of class III 18-23 might single out PylRS variants that accept bulky ncAAs, since the volume of $\mathrm{CF}_{3}$ substituent $\left(39.8 \AA^{3}\right)$ is about twice as big as a methyl-group $\left(21.6 \AA^{3}\right)^{26}$.

Pocket-substrate relationship. As expected, the 20 incorporated ncAAs required diverse structural adaptions of PylRS (Fig. 4). Y271 (P1) defines the bottom of the binding pocket; therefore, this position strongly varied with the ncAA size. While tyrosine was highly conserved at P1 for the short ncAAs 1, 2, 10, 11, and 14 alanine was favored by the larger ncAAs 4-7, 13, 15-17, 19, and 20. Aminoacylation of the longest ncAAs 8 and 21 was preferred by glycine at P1 of the active site. We observed a similar trend for the position L274 (P2), which constitutes the rear end of the binding pocket. While leucine was favored by the small ncAAs 1, 10, and 11, the shorter valine occurred in high abundance for larger ncAAs. Interestingly, the mutation C313V (P3), was strictly conserved throughout all sequenced variants. M315 (P4) differed widely for small ncAAs; however, tyrosine was more abundant among the larger ncAAs 8, 16, 20, and 21. V370 (P5) and I378 (P6) were not influenced by the size or chemistry of the incorporated ncAAs. Arginine and isoleucine respectively appeared with the highest frequency at these positions.

Design of a polyspecific PyIRS variant. We combined potential key mutations Y271A, L274V, C313V, M315Y, Y349F, and V370R to design a highly polyspecific PylRS (HpRS) for the incorporation of large ncAAs. To test the substrate scope, the HpRS/tRNA ${ }^{\text {Pyl }}$ pair and mTFP $_{\text {TAG }_{28}}$ were expressed with 1-35 in E. coli. The incorporation of the ncAAs 1-8, 10-21, 24-26, 28-35 was detected by fluorescence signal (Fig. 5) and additionally confirmed by SDS-PAGE (Supplementary Fig. 1) and ESI-TOF (Supplementary Table 4, Supplementary Fig. 2). Further, we used Alexa Fluor 532 streptavidin conjugate to confirm the incorporation of ncAA 35 in mTFP $_{\text {TAG128 }}$ (Supplementary Fig. 3).

Intriguingly, HpRS tolerated a broad range of lysine carbamates with diverse features, including aliphatic chains, fluorinated residues, cyclic structures, and a biotinylated ncAA. As predicted, HpRS was highly capable of aminoacylation of ncAAs with long side chains 3-6; yet, the smallest tested ncAAs were only moderately accepted (Fig. 5). Therefore, the mutations $\mathrm{C} 313 \mathrm{~V}$ and $\mathrm{Y} 349 \mathrm{~F}$ were combined based on the heat map to generate a PylRS particular suited for small ncAAs. Indeed, this variant showed a significant higher aminoacylation efficiency of the small ncAAs 1, 10, and 11 than HpRS (Fig. 6D).

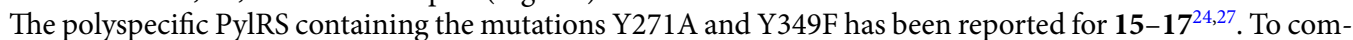
pare HpRS with PylRS (Y271A, Y349F), both synthetases were co-expressed with $\mathrm{mTFP}_{\mathrm{TAG} 128}$ in the presence of 15-17 and the fluorescence signals were analysed. The ncAAs were incorporated in $\mathrm{mTFP}_{\mathrm{TAG} 128}$ in equivalent yields by both synthetases (Supplementary Fig. 4).

Labeling of nanobodies. Immunoassays such as flow cytometry or western blot usually require commercially available antibodies-conjugates. In contrast, nanobodies derived from Camelid antibodies, offer nowadays 


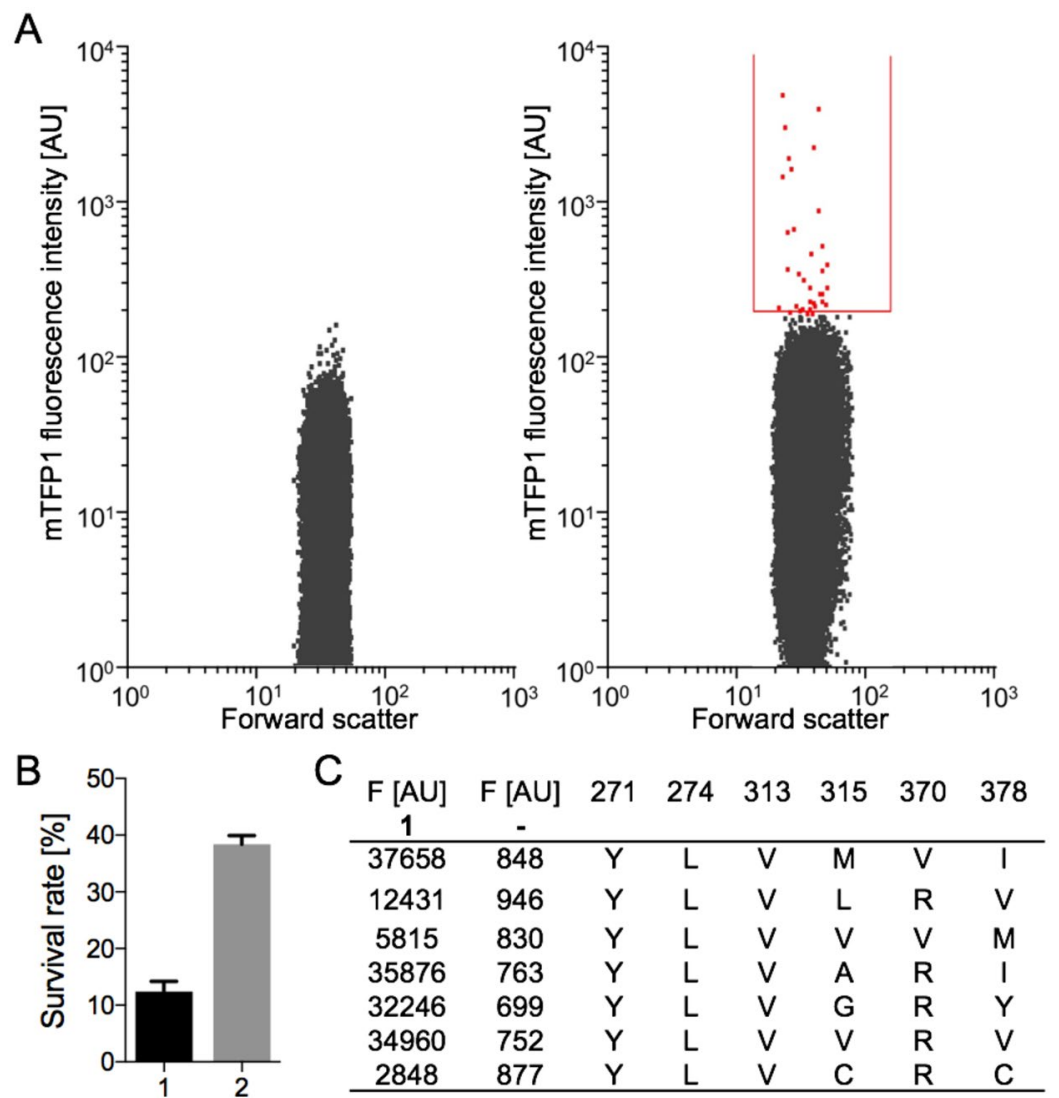

Figure 3. Identification of PylRS variants for the incorporation of ncAA 1. (A) (Left plot) Fluorescence intensity and forward scatter of E. coli cells harboring pEVOL303_Lib in the absence of ncAAs analyzed by FACS. (Right plot) Cells harboring pEVOL303_Lib were grown in the presence of $\mathbf{1}$. Fluorescent cells (red) within the sort gate (red line) were separated from nonfluorescent cells. (B) Survival rate of cells after FACS in LB medium (1) and conditioned medium (2). Results were obtained from five independent experiments; standard deviation is indicated by error bars. (C) Fluorescence intensities of mTFP $1_{\text {TAG128 }}$ expressed with 1 or without - in the present of seven different PylRS variants (mutations are shown).

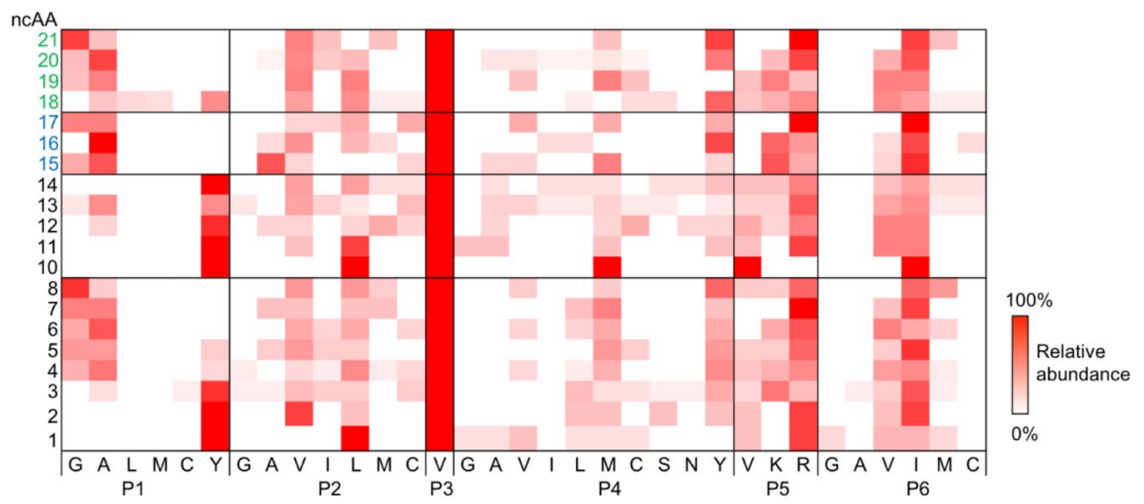

Figure 4. Heat map of the analyzed ncAAs 1-23 versus the six variable positions of the PylRS library $(\mathrm{P} 1=\mathrm{Y} 271, \mathrm{P} 2=\mathrm{L} 274, \mathrm{P} 3=\mathrm{C} 313, \mathrm{P} 4=\mathrm{M} 315, \mathrm{P} 5=\mathrm{V} 370, \mathrm{P} 6=\mathrm{I} 378)$. Amino acids are represented by a oneletter code; the color intensity indicates the relative abundance of a mutation. Mutations not present in any of the sequenced PylRS variants have been omitted form the map. The ncAAs 9, 22, and 23 were not incorporated.

an attractive alternative ${ }^{28}$, as they are highly stable, small and can be expressed in heterologous systems such as E. coli in high yields ${ }^{29}$. The developed HpRS/tRNA ${ }^{\text {Pyl }}$ pair was employed to incorporate ncAA 14, 17, 35 in an anti-GFP nanobody, which exhibits a specific binding activity against the green fluorescence protein (GFP) $)^{30}$, at the exposed position $\mathrm{K} 44$ with expression yields of $8 \mathrm{mg} / \mathrm{L}, 4 \mathrm{mg} / \mathrm{L}, 4 \mathrm{mg} / \mathrm{L}$ in E. coli culture, respectively. The 
A

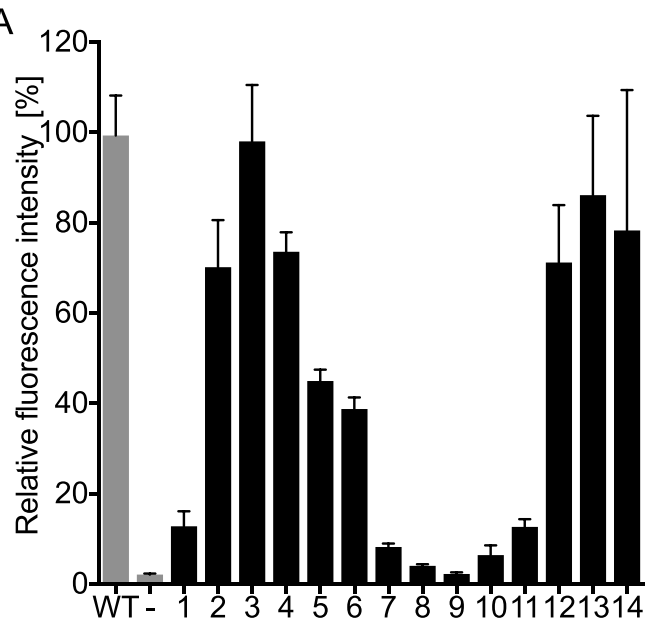

C

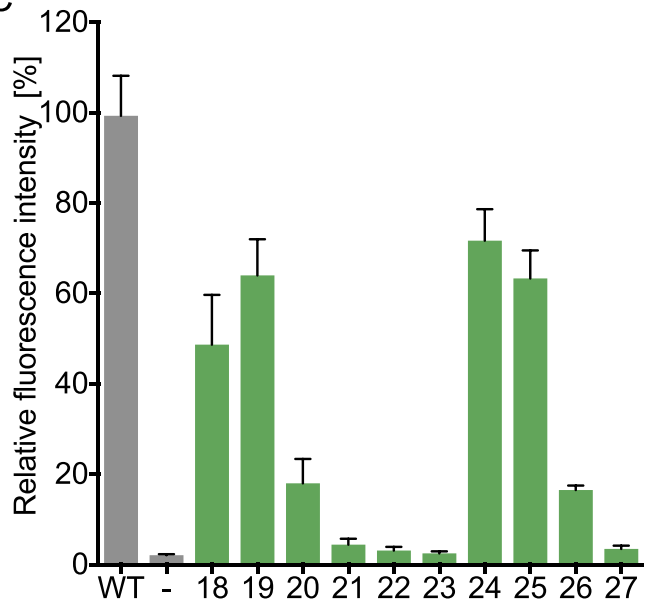

B

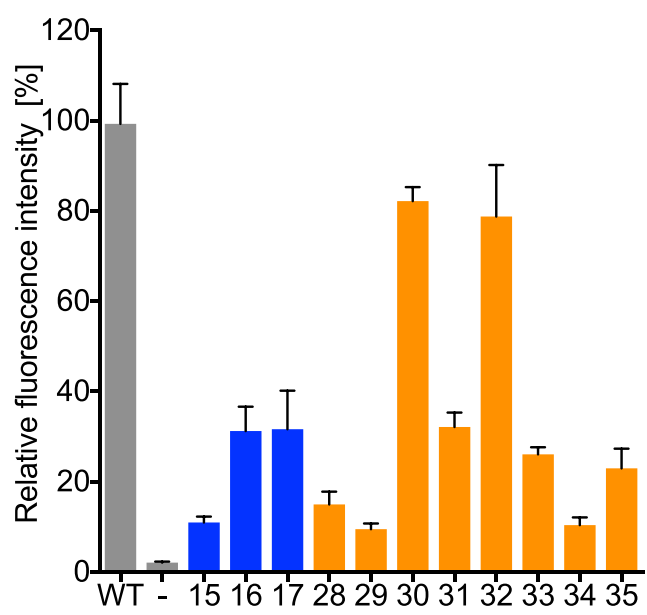

$\mathrm{D}$

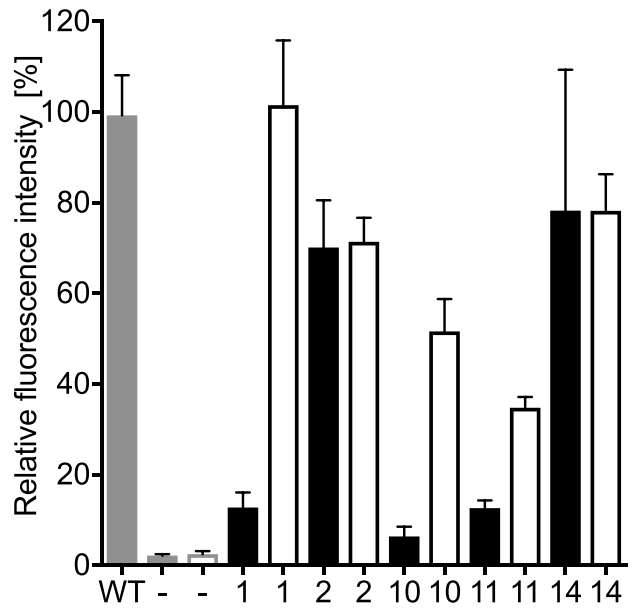

Figure 5. HpRS-dependent incorporation of ncAAs 1-35 in mTFP $1_{\text {TAG128. }}$. Fluorescence intensity of mTFP1 is normalized against wild-type (WT) mTFP1 (grey bar), while expression of mTFP1 $1_{\text {TAG128 }}$ in the absence of ncAAs (-, grey bar) served as a negative control. Error bars indicate the standard deviation of three independent experiments. (A) Substrate profiles of HpRS for class I (black): ncAAs 1-14. (B) Class II (blue): ncAAs 15-17 and class IV (orange): ncAAs 28-35. (C) Class III (green): ncAAs 18-27. (D) Comparison of the aminoacylation efficiencies of HpRS (solid) and the PylRS variant (C313V, Y349F) (no fill) for ncAAs 1, 2, 10, 11 , and 14 .

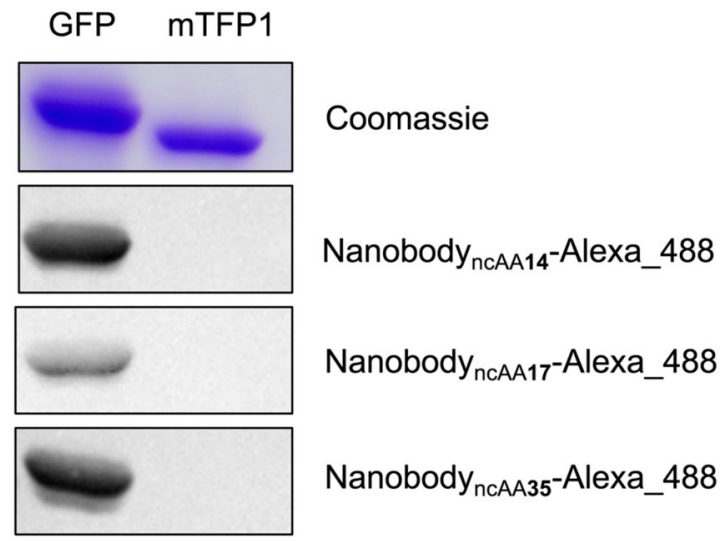

Figure 6. Coomassie stained SDS gel of cell lysates containing GFP or mTFP1 and western blot on the same cell lysates with an anti-GFP nanobody bearing ncAA 14, 17 or 35 conjugated to Alexa Fluor 488. 
ncAA incorporation into anti-GFP nanobody $\mathrm{TAG}_{44}$ was confirmed by SDS-PAGE (Supplementary Fig. 5) and LC-MS analysis. To conjugate the anti-GFP nanobody ${ }_{\text {ncAA14 }}$, the applied azide was linked to an Alexa Fluor 488 alkyne via copper-catalyzed azide-alkyne "click" reaction. The bicyclononyne-lysine (BCN), ncAA 17, in the anti-GFP nanobody ${ }_{\text {ncAA17 }}$ formed a strain-promoted [3+2] azide-alkyne cycloaddition with an Alexa Fluor 488 azide. The biotin containing anti-GFP nanobody ${ }_{\text {ncAA35 }}$ bound successfully an Alexa Fluor 488 marked streptavidin.

A western blot of cell lysates containing GFP or mTFP1, as a negative control, demonstrated the specific detection of GFP by the established anti-GFP nanobody-conjugates (Fig. 6). Notably, the most efficient coupling reaction between anti-GFP nanobody and fluorescence-dye seemed to be the copper-catalyzed "click" reaction and the biotin-streptavidin interaction, thus resulting in the strongest western blot signal (Fig. 6).

In summary, we demonstrated that the PylRS-substrate interaction can be used to recombine key mutation to generate new PylRS variants. This seems at first glance a limiting engineering approach, however as a proof of concept the designed HpRS exhibit a remarkable substrate scope and facilitates the incorporation of a biotin bearing ncAA. A prerequisite for this method is the generation of a diverse pool of beneficial PylRS variants that was achieved by a fast and mild FACS setup. PylRS diversity for a particular ncAA provided insights into the complex PylRS-substrate interaction and allowed the prediction of polyspecific variants and can now be used as an example for others to avoid the cumbersome engineering process.

Moreover, HpRS was used to insert 14, 17 or 35 in an anti-GFP nanobody. The three different chemical handles were exploited to produce fluorescent nanobody-conjugates. In particular, the streptavidin-biotin interaction allows efficient labeling of the anti-GFP nanobody without a catalyst. Therefore, the incorporation of $\mathbf{3 5}$ might be a good starting point to simplify techniques such as western blotting, ELISA, flow cytometry or antibody labeling in general.

\section{Methods}

Strains. All cloning steps were performed in TOP10 E. coli (Thermo Fisher Scientific). The FACS and protein expression experiments were carried out with BL21 (DE3) (Lucigen, Middleton, WI).

Medium. A conditioned medium was used to improve the survival of sorted cells after FACS. To prepare the conditioned medium, BL21 (DE3) cultures were grown in $\mathrm{LB}\left(\mathrm{OD}_{600} \sim 1.0\right)$, centrifuged at $8000 \times \mathrm{g}$ for $20 \mathrm{~min}$ at $4^{\circ} \mathrm{C}$ and the supernatant was sterilized by passing through a $0.22 \mu \mathrm{m}$ cellulose filter (Millipore, Bedford, MA).

Non-canonical amino acids (ncAA). ncAA 1 (Sigma-Aldrich), ncAA 15-17 (Sirius Fine Chemicals, Bremen, Germany), and ncAAs 24-27, 30-33, as well as 35 (SUNGYOUNG Chemical Limited, Shanghai, China) were purchased from commercial sources. ncAA 34 was synthesized according to the procedure published by Luo et al. ${ }^{31}$ ncAA $\mathbf{2 - 1 4}, \mathbf{1 8 - 2 3}, \mathbf{2 8}$, and 29 were synthesized according to a modified procedure from Li et al. ${ }^{32}$ (Supplementary Information, S2).

Plasmid construction. The plasmid pEVOL303 was constructed by ligating the plasmid $\mathrm{pEVOL}^{33}$ with the commercially available pET303/CT-His (Thermo Fisher Scientific) using Gibson cloning ${ }^{34}$. Prior to ligation, pEVOL was digested with the restriction enzymes SacI and PciI. The required region of pET303 was amplified using the primers pET303_f and pET303_r (Supplementary Table 2). The resulting plasmid, pEVOL303, carried a p15A origin, a chloramphenicol resistance gene, a Methanosarcina barkeri PylRS gene downstream of a pBAD promoter, and the corresponding $t R N A$ gene controlled by a proK promoter. The fluorescent protein gene with an $\mathrm{N}$-terminal 6xHis-SUMO-tag, either wild-type mTFP1 or mTFP1 $1_{\text {TAG128 }}$ (bearing an amber codon at position 128), was cloned downstream of a T7 promoter. The amber codon was inserted using the primers mTFP_128_f and mTFP_128_r (Supplementary Table 2). The GFP-nanobody was encoded on the plasmid pOPINE ${ }^{30}$. The amber codon was inserted at position 44 using the primers GFP_Nano_f and GFP_Nano_r (Supplementary Table 2).

PyIRS library construction. Methanosarcina barkeri. PylRS containing the single mutation Y349F served as the starting point for our mutant library. Six positions in PylRS, Y271, L274, C313, M315, V370, and I378, were selected for our library based on a homology model derived from the crystal structure of PylRS (PDB 4Q6G and 4CS3) (Fig. 1A,B) ${ }^{35,36}$. A pre-defined set of amino acids was inserted into the six positions, resulting in 95,040 mutants. To ensure an equal distribution of all the mutants, the library was assembled non-codon redundant and codon usage optimized by the company, Life Technologies (Thermo Fisher Scientific) and introduced to pEVOL303 via the restriction sites SalI and BglII. The resulting plasmid, pEVOL303_Lib, was transformed into BL21 (DE3) cells by applying the standard electroporation protocol. The transformation was determined with $1 \times 10^{7} \mathrm{CFU}$ per preparation.

Sample preparation for FACS. BL21 (DE3) cells transformed with pEVOL303_Lib were inoculated in $50 \mathrm{~mL}$ LB medium supplemented with $25 \mu \mathrm{g} / \mathrm{mL}$ chloramphenicol $(\mathrm{Cm})$, and grown until an $\mathrm{OD}_{600}$ of 0.7 . Then, $450 \mu \mathrm{L}$ of the cells was transferred into a $2 \mathrm{~mL}$ reaction vessel and induced with $50 \mu \mathrm{L}$ induction medium $(10 \mathrm{mM}$ ncAA, $10 \mathrm{mM}$ IPTG, $1 \% \mathrm{w} / \mathrm{v}$ arabinose) and incubated for $16 \mathrm{~h}$ at $37^{\circ} \mathrm{C}$ and $700 \mathrm{rpm}$. The cells were diluted to a final concentration of $1 \times 10^{7}$ cell $/ \mathrm{mL}$ and washed twice with an $\mathrm{M} 9$ minimal medium supplemented with $25 \mu \mathrm{g} /$ $\mathrm{mL}$ chloramphenicol. In the beginning of each FACS experiment, the negative control (cell growth in the absence of ncAA) was screened first to ensure the discrimination of canonical amino acids by the PylRS mutant library. Cell sorting was performed by detecting the fluorescence of mTFP1 with a BD Influx (BD Biosciences) operated with filter-sterilized BD FACS Flow Sheath Fluid, a $457 \mathrm{~nm}$ laser for excitation, and a 480/40 bandpass filter. The selected operation mode was 1.0 Drop Single. The selection threshold (gate) was adjusted based on the fluorescent signal from the first $1 \times 10^{5}$ cells of each sample. Cells within the gate were sorted separately on a 96 -well plate (Sigma-Aldrich) supplied with the conditioned medium containing $25 \mu \mathrm{g} / \mathrm{mL}$ chloramphenicol. The growth of 
the sorted cells was continued overnight at $37^{\circ} \mathrm{C}$ and $300 \mathrm{rpm}$. Each culture was sequenced using the primers Lib_seq_f and Lib_seq_r (Supplementary Table 2).pEVOL303_Lib containing the sequenced PylRS variants were retransformed into new BL21 (DE3) cells, grown and induced with the induction medium at $\mathrm{OD}_{600}$ of 0.7 . Correspondingly, PylRS, tRNA ${ }^{\mathrm{Pyl}}$, and $\mathrm{mTFP}_{\mathrm{TAG} 128}$ were expressed. Both the incorporation of the ncAAs and the orthogonality of the PylRS variant were confirmed by fluorescence measurement of mTFP1 (excitation $462 \mathrm{~nm}$, emission $492 \mathrm{~nm}$ ) in a black, 96-well plate (Thermo Fisher Scientific) with an Infinite M1000 plate reader (Tecan, Zurich, Switzerland).

ncAA incorporation. A single BL21 (DE3) colony containing pEVOL303 encoding for HpRS, PylRS (Y271A and Y349F) or PylRS (C313V and Y349F) was selected from the $\mathrm{LB} / \mathrm{Cm}$ agar plate and inoculated into $50 \mathrm{~mL} \mathrm{LB} /$ $\mathrm{Cm}$ media, then incubated and shaken overnight at $37^{\circ} \mathrm{C}$. The resulting culture was diluted $(1: 100)$ with fresh $\mathrm{LB} / \mathrm{Cm}$ media and grown to an $\mathrm{OD}_{600}$ of 1.5 . The mTFP1 expression was triggered by adding $1 \mathrm{mM} \mathrm{ncAA}, 1 \mathrm{mM}$ IPTG, and $0.1 \% \mathrm{w} / \mathrm{v}$ arabinose and cell growth continued at $37^{\circ} \mathrm{C}$ and $700 \mathrm{rpm}$ for $16 \mathrm{~h}$. Fluorescence was measured correspondingly. For SDS-PAGE, the sample was heated at $75^{\circ} \mathrm{C}$ for $20 \mathrm{~min}$ and cleared by centrifugation. For protein purification, cell pellet was suspended in lysis buffer $(100 \mathrm{mM}$ Tris pH 7.5, $500 \mathrm{mM} \mathrm{NaCl}, 20 \mathrm{mM}$ imidazole, $10 \%$, v/v glycerol), incubated at $75^{\circ} \mathrm{C}$ for $20 \mathrm{~min}$ and cleared by centrifugation

The supernatant was loaded onto a HisTrap HP Ni-NTA column (GE Healthcare). Protein eluted by increasing the concentration of imidazole from $20 \mathrm{mM}$ to $500 \mathrm{mM}$ in the lysis buffer over 10 column volumes. SUMO protease was added to the eluted protein, dialyzed overnight at $4{ }^{\circ} \mathrm{C}$ against $\mathrm{ddH}_{2} \mathrm{O}$ and passed through a HisTrap HP Ni-NTA to remove the remaining N-terminal 6xHis- as well as the SUMO-tag.

The HpRS/tRNA ${ }^{\text {Pyl }}$ pair was employed to incorporate the respective ncAAs at position $\mathrm{K} 44$ of the reported anti-GFP nanobody ${ }^{30}$. BL21(DE3) were grown at $37^{\circ} \mathrm{C}$ to $\mathrm{OD}_{600} 0.7$ in $500 \mathrm{~mL} \mathrm{LB}$ medium. Protein synthesis was induced by $0.5 \mathrm{mM}$ IPTG, $0.1 \% \mathrm{w} / \mathrm{v}$ arabinose, and $1 \mathrm{mM} \mathrm{ncAA}$. Propagation was carried out at $20^{\circ} \mathrm{C}$ for $20 \mathrm{~h}$. Cells were harvested by centrifugation, lysed by sonication and cleared by centrifugation. The cell lysate was applied onto a HisTrap HP Ni-NTA column and eluted in $50 \mathrm{mM}$ Tris- $\mathrm{HCl} \mathrm{pH} \mathrm{7.5,300} \mathrm{mM} \mathrm{NaCl,} 1 \mathrm{mM}$ TCEP, $500 \mathrm{mM}$ Imidazole. The eluted protein was concentrated and loaded onto a HiLoad Superdex 200 (16/60) size-exclusion column (GE Healthcare) equilibrated with $50 \mathrm{mM}$ Tris- $\mathrm{HCl} \mathrm{pH} \mathrm{7.5,} 150 \mathrm{mM} \mathrm{NaCl}, 1 \mathrm{mM}$ TCEP.

Conjugation of nanobodies. $500 \mathrm{nM}$ purified GFP-nanobody containing 14, 17 or 35 was labeled with either Alexa 488 alkyne $(40 \mu \mathrm{M}, 1 \mathrm{~h})$ using the Click-iT Protein Reaction Buffer Kit, Alexa 488 azide $(40 \mu \mathrm{M}, 6 \mathrm{~h})$ or Alexa 488 streptavidin $(5 \mu \mathrm{M}, 1 \mathrm{~h})$ at $37^{\circ} \mathrm{C}$ (Thermo Fisher Scientific).

Western blot. E. coli cell lysate containing GFP or mTFP1 was electrophoretically fractionated and electroblotted onto $0.45 \mu \mathrm{m}$ Immobilon-P polyvinylidene difluoride (PVDF) membranes (Merck, Darmstadt, Germany). After electroblotting, the membranes were washed three times for $5 \mathrm{~min}$ with TBS buffer and blocked in TBST $(10 \mathrm{mM}$ Tris, $0.9 \%, \mathrm{w} / \mathrm{v} \mathrm{NaCl}, 0.05 \%, \mathrm{v} / \mathrm{v}$, Tween-20, $\mathrm{pH} 7.4)$ with $1 \%(\mathrm{w} / \mathrm{v})$ casein for $3 \mathrm{~h}$ at room temperature. The blots were probed and incubated with the conjugated GFP-nanobodies $(1 \mu \mathrm{g} / \mathrm{mL})$ at room temperature for $60 \mathrm{~min}$. The washing steps were performed with TBST. Fluorescence was detected in an iBright FL1000 imaging system (Invitrogen).

Mass spectrometry. Pure mTFP1 and GFP-nanobodies were analysed by mass spectrometry (maXis HD ESI-TOF, Bruker). The sample was injected into a high-performance liquid chromatography (Agilent Technologies, C4 column, column volume of $5 \mathrm{~mL}$ ) and separation was performed at a constant flow rate of $0.5 \mu \mathrm{L} / \mathrm{min}$ and a gradient of $80 \%$ acetonitrile and $0.1 \%$ formic acid for $8 \mathrm{~min}$. Fractions were recorded according to the standard procedure.

Homology model of the Methanosarcina barkeri PyIRS in complex with pyrrolysine. Homology

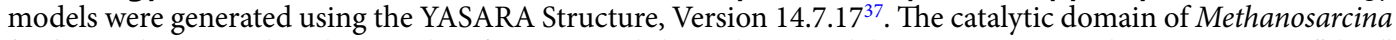
barkeri PylRS served as the template for YASARA's homology modeling macro, using the conservative "slow" protocol with the following parameter settings: number of PSI-BLAST iterations - 10; maximum allowed BLAST E-value to consider template - 0.5 ; maximum oligomerization state - 2 ; maximum number of alignment variations per template - 4; maximum number of conformations tried per loop - 200; and maximum number of residues added to the termini -20 . The resulting homology model was based on two structures of PylRS variants, PDB 4Q6G and 4CS3; both sequences had a 98\% homology with the template ${ }^{35,36}$. The YASARA algorithm performed the secondary structure prediction, loop construction, and amino-acid rotamer selection, followed by a steepest-descent energy minimization.

\section{References}

1. Neumann, H., Peak-Chew, S. Y. \& Chin, J. W. Genetically encoding N(epsilon)-acetyllysine in recombinant proteins. Nat. Chem. Biol. 4, 232-234, https://doi.org/10.1038/nchembio.73 (2008).

2. Liu, C. C. \& Schultz, P. G. Adding new chemistries to the genetic code. Annu. Rev. Biochem. 79, 413-444, https://doi.org/10.1146/ annurev.biochem.052308.105824 (2010).

3. Lang, K. et al. Genetic encoding of bicyclononynes and trans-cyclooctenes for site-specific protein labeling in vitro and in live mammalian cells via rapid fluorogenic Diels-Alder reactions. J. Am. Chem. Soc. 134, 10317-10320, https://doi.org/10.1021/ ja302832g (2012).

4. Plass, T. et al. Amino acids for Diels-Alder reactions in living cells. Angew. Chem. Int. Ed. Engl. 51, 4166-4170, https://doi. org/10.1002/anie.201108231 (2012).

5. Dumas, A., Lercher, L., Spicer, C. D. \& Davis, B. G. Designing logical codon reassignment - Expanding the chemistry in biology. Chem. Sci. 6, 50-69, https://doi.org/10.1039/c4sc01534g (2015).

6. Wang, L., Brock, A., Herberich, B. \& Schultz, P. G. Expanding the genetic code of Escherichia coli. Science 292, 498-500, https://doi. org/10.1126/science.1060077 (2001) 
7. Fekner, T. \& Chan, M. K. The pyrrolysine translational machinery as a genetic-code expansion tool. Curr. Opin. Chem. Biol. 15, 387-391, https://doi.org/10.1016/j.cbpa.2011.03.007 (2011).

8. Wan, W., Tharp, J. M. \& Liu, W. R. Pyrrolysyl-tRNA synthetase: an ordinary enzyme but an outstanding genetic code expansion tool. Biochim. Biophys. Acta. 1844, 1059-1070, https://doi.org/10.1016/j.bbapap.2014.03.002 (2014).

9. Polycarpo, C. R. et al. Pyrrolysine analogues as substrates for pyrrolysyl-tRNA synthetase. FEBS Lett. 580, 6695-6700, https://doi. org/10.1016/j.febslet.2006.11.028 (2006).

10. Yanagisawa, T. et al. Multistep engineering of pyrrolysyl-tRNA synthetase to genetically encode N(epsilon)-(oazidobenzyloxycarbonyl) lysine for site-specific protein modification. Chem. Biol. 15, 1187-1197, https://doi.org/10.1016/j. chembiol.2008.10.004 (2008).

11. Hancock, S. M., Uprety, R., Deiters, A. \& Chin, J. W. Expanding the genetic code of yeast for incorporation of diverse unnatural amino acids via a pyrrolysyl-tRNA synthetase/tRNA pair. J. Am. Chem. Soc. 132, 14819-14824, https://doi.org/10.1021/ja104609m (2010).

12. Xie, J. \& Schultz, P. G. A chemical toolkit for proteins-an expanded genetic code. Nat. Rev. Mol. Cell. Biol. 7, 775-782, https://doi. org $/ 10.1038 / \mathrm{nrm} 2005$ (2006).

13. Voloshchuk, N. \& Montclare, J. K. Incorporation of unnatural amino acids for synthetic biology. Mol. Biosyst. 6, 65-80, https://doi. org/10.1039/b909200p (2010).

14. Wang, L., Zhang, Z., Brock, A. \& Schultz, P. G. Addition of the keto functional group to the genetic code of Escherichia coli. Proc. Natl. Acad. Sci. 100, 56-61, https://doi.org/10.1073/pnas.0234824100 (2003).

15. Santoro, S. W., Wang, L., Herberich, B., King, D. S. \& Schultz, P. G. An efficient system for the evolution of aminoacyl-tRNA synthetase specificity. Nat. Biotechnol. 20, 1044-1048, https://doi.org/10.1038/nbt742 (2002).

16. Link, A. J. et al. Discovery of aminoacyl-tRNA synthetase activity through cell-surface display of noncanonical amino acids. Proc. Natl. Acad. Sci. 103, 10180-10185, https://doi.org/10.1073/pnas.0601167103 (2006).

17. Kuhn, S. M., Rubini, M., Fuhrmann, M., Theobald, I. \& Skerra, A. Engineering of an orthogonal aminoacyl-tRNA synthetase for efficient incorporation of the non-natural amino acid O-methyl-L-tyrosine using fluorescence-based bacterial cell sorting. J. Mol. Biol. 404, 70-87, https://doi.org/10.1016/j.jmb.2010.09.001 (2010).

18. Owens, A. E., Grasso, K. T., Ziegler, C. A. \& Fasan, R. Two-tier screening platform for directed evolution of aminoacyl-tRNA synthetases with enhanced stop codon suppression efficiency. Chembiochem 18, 1109-1116, https://doi.org/10.1002/cbic.201700039 (2017).

19. Miyake-Stoner, S. J. et al. Generating permissive site-specific unnatural aminoacyl-tRNA synthetases. Biochemistry 49, 1667-1677, https://doi.org/10.1021/bi901947r (2010).

20. Cooley, R. B., Karplus, P. A. \& Mehl, R. A. Gleaning unexpected fruits from hard-won synthetases: probing principles of permissivity in non-canonical amino acid-tRNA synthetases. Chembiochem 15, 1810-1819, https://doi.org/10.1002/cbic.201402180 (2014).

21. Kavran, J. M. et al. Structure of pyrrolysyl-tRNA synthetase, an archaeal enzyme for genetic code innovation. Proc. Natl. Acad. Sci. 104, 11268-11273, https://doi.org/10.1073/pnas.0704769104 (2007).

22. Wang, Y. S. et al. The de novo engineering of pyrrolysyl-tRNA synthetase for genetic incorporation of L-phenylalanine and its derivatives. Mol. Biosyst. 7, 714-717, https://doi.org/10.1039/c0mb00217h (2011).

23. Yoo, T. H. \& Tirrell, D. A. High-throughput screening for methionyl-tRNA synthetases that enable residue-specific incorporation of noncanonical amino acids into recombinant proteins in bacterial cells. Angew. Chem. Int. Ed. Engl. 46, 5340-5343, https://doi. org/10.1002/anie.200700779 (2007).

24. Nikic, I. et al. Minimal tags for rapid dual-color live-cell labeling and super-resolution microscopy. Angew. Chem. Int. Ed. Engl. 53, 2245-2249, https://doi.org/10.1002/anie.201309847 (2014).

25. Lang, K. \& Chin, J. W. Cellular incorporation of unnatural amino acids and bioorthogonal labeling of proteins. Chem. Rev. 114, 4764-4806, https://doi.org/10.1021/cr400355w (2014).

26. Muller, K., Faeh, C. \& Diederich, F. Fluorine in pharmaceuticals: looking beyond intuition. Science 317, 1881-1886, https://doi. org/10.1126/science.1131943 (2007).

27. Plass, T., Milles, S., Koehler, C., Schultz, C. \& Lemke, E. A. Genetically encoded copper-free click chemistry. Angew. Chem. Int. Ed. Engl. 50, 3878-3881, https://doi.org/10.1002/anie.201008178 (2011).

28. De Meyer, T., Muyldermans, S. \& Depicker, A. Nanobody-based products as research and diagnostic tools. Trends. Biotechnol. 32, 263-270, https://doi.org/10.1016/j.tibtech.2014.03.001 (2014).

29. Muyldermans, S. Nanobodies: natural single-domain antibodies. Annu. Rev. Biochem. 82, 775-797, https://doi.org/10.1146/ annurev-biochem-063011-092449 (2013).

30. Kubala, M. H., Kovtun, O., Alexandrov, K. \& Collins, B. M. Structural and thermodynamic analysis of the GFP:GFP-nanobody complex. Protein Sci. 19, 2389-2401, https://doi.org/10.1002/pro.519 (2010).

31. Luo, J. et al. Genetically encoded optochemical probes for simultaneous fluorescence reporting and light activation of protein function with two-photon excitation. J. Am. Chem. Soc. 136, 15551-15558, https://doi.org/10.1021/ja5055862 (2014).

32. Li, H. M., Chen, C. Y. \& Padros, J. B. Highly efficient carbamate formation from alcohols and hindered amino acids or esters using N,N'-Disuccinimidyl Carbonate (DSC). Synlett 2011, 1454-1458, https://doi.org/10.1055/s-0030-1260584 (2011).

33. Young, T. S., Ahmad, I., Yin, J. A. \& Schultz, P. G. An enhanced system for unnatural amino acid mutagenesis in E. coli. J. Mol. Biol. 395, 361-374, https://doi.org/10.1016/j.jmb.2009.10.030 (2010).

34. Gibson, D. G. et al. Enzymatic assembly of DNA molecules up to several hundred kilobases. Nat. Methods 6, 343-345, https://doi. org/10.1038/nmeth.1318 (2009).

35. Guo, L. T. et al. Polyspecific pyrrolysyl-tRNA synthetases from directed evolution. Proc. Natl. Acad. Sci. 111, 16724-16729, https:// doi.org/10.1073/pnas.1419737111 (2014).

36. Schmidt, M. J., Weber, A., Pott, M., Welte, W. \& Summerer, D. Structural basis of furan-amino acid recognition by a polyspecific aminoacyl-tRNA-synthetase and its genetic encoding in human cells. Chembiochem 15, 1755-1760, https://doi.org/10.1002/ cbic.201402006 (2014).

37. Krieger, E. \& Vriend, G. New ways to boost molecular dynamics simulations. J. Comput. Chem. 36, 996-1007, https://doi. org/10.1002/jcc.23899 (2015).

\section{Acknowledgements}

The research reported in this publication was supported by funding from King Abdullah University of Science and Technology (KAUST). We thank the SFB749/A10 (M.G.) for financial support. We are grateful to Prof. Peter G. Schultz (The Scripps Research Institute, La Jolla, CA) for kindly providing the original pEVOL-PylRS plasmid.

\section{Author Contributions}

J.E. and M.R. conceived and supervised the study; A.H. and R.K. designed the experiments; A.H., R.K., A.A., D.R. and X.L. performed experiments, A.H., R.K., S.G. and M.G. analyzed data; A.H. and R.K. wrote the manuscript; A.H., R.K., M.G., M.R. and J.E. made manuscript revisions. 


\section{Additional Information}

Supplementary information accompanies this paper at https://doi.org/10.1038/s41598-019-48357-0.

Competing Interests: The authors declare the following competing financial interest(s): Patent filed with the United States Patent and Trademark Office (USPTO) on the May 08, 2017, and assigned serial number 62/502, 860.

Publisher's note: Springer Nature remains neutral with regard to jurisdictional claims in published maps and institutional affiliations.

(c) Open Access This article is licensed under a Creative Commons Attribution 4.0 International License, which permits use, sharing, adaptation, distribution and reproduction in any medium or format, as long as you give appropriate credit to the original author(s) and the source, provide a link to the Creative Commons license, and indicate if changes were made. The images or other third party material in this article are included in the article's Creative Commons license, unless indicated otherwise in a credit line to the material. If material is not included in the article's Creative Commons license and your intended use is not permitted by statutory regulation or exceeds the permitted use, you will need to obtain permission directly from the copyright holder. To view a copy of this license, visit http://creativecommons.org/licenses/by/4.0/.

(c) The Author(s) 2019 